
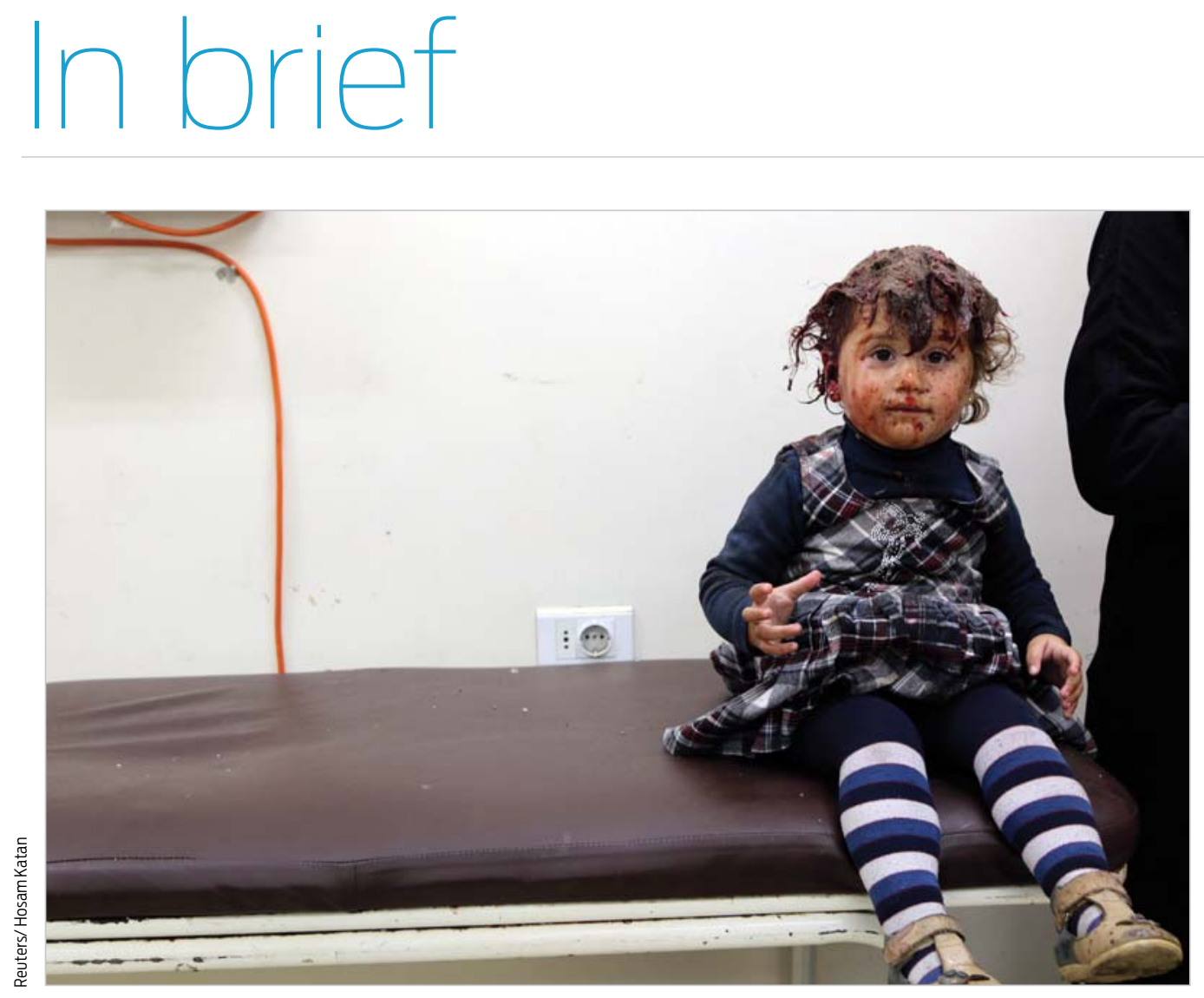

An injured girl waits for treatment at a clinic after being injured by what activists said were barrel bombs, dropped in Aleppo's al-Sakhour district by forces loyal to Syria's President Bashar al-Assad.

\title{
From the NHMRC
}

\section{What's the evidence?}

Reaction to the recently released National Health and Medical Research Council (NHMRC) draft information paper, Evidence on wind farms and human health (http://consultations. nhmrc.gov.au/files/consultations/ drafts/nhmrcdraftinformationpaper publicconsultationfebruary2014.pdf), raises interesting issues about public perceptions of research, evidence, and cause and effect.

The NHMRC believes it would be useful to describe its methods for examining an area of considerable public interest.

First, a commissioned independent group of experts completes a systematic review of the available research literature, using a defined protocol and search strategy, so that all relevant publications are included and the scientific rigour of each study is considered.

The NHMRC then establishes an advisory group (whose members' interests are published on the
Council website), which develops an information paper based on the evidence in the review. This advisory group grades the body of evidence and develops a document for consideration by the NHMRC.

Next, the Council releases a draft information paper or position statement for public consultation and considers all feedback from the consultation process. This practice is in line with section 3 of the National Health and Medical Research Council Act 1992, which states: "to the extent that it is practicable to do so, the NHMRC should adopt a policy of public consultation in relation to individual and public health matters being considered by it". Not only is community input required by law, but it also adds significant value.

The review undertaken for the draft information paper on wind farms and human health identified and evaluated nearly 3000 publications, including individual pieces of research, reviews and commentary. We also called for

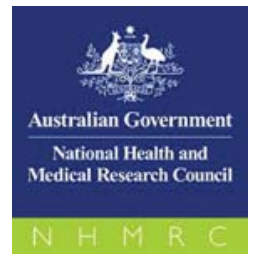

public submissions and received around 500 of these. Community consultation closed on 11 April 2014. Next, we will release a final information paper, and it is likely that we will issue a position statement later in 2014.

It has been our repeated experience that the cherry-picking of evidence by vested interests is common in public health matters. This can unbalance the public debate, which is often passionate about public health. Our role is to dispassionately draw conclusions from the rigorous, unbiased analysis of all available evidence and its quality.

The wind farm information paper states: "There is no reliable or consistent evidence that wind farms directly cause adverse health effects in humans". This is a straightforward factual statement of the level of currently available evidence on the topic; however, it is interpreted differently by stakeholders in the doi: 10.5694/mjal4.00362 surrounding debate. 


\section{Kawasaki disease incidence three times higher}

The likely incidence of Kawasaki disease (KD) in Australia is almost three times higher than previously estimated, according to research published in Pediatrics. Using 30 years of Western Australian data, the authors found that the mean annual incidence of KD increased from 2.82 per 100000 children aged under 5 years in 1980-1989 to 7.96 in 1990-1999 and 9.34 in 2000-2009, mirroring results in other industrialised European-Caucasian populations. The increase, the authors wrote, "likely reflects both improved ascertainment and a real increase in disease burden". When extrapolated to the national population, the authors estimated that there were approximately "170 to 180 " patients with KD hospitalised each year. Coronary artery involvement was "relatively high" at $23.4 \%$ and was more prevalent in boys.

Pediatrics 2014; 31 March (online). doi: 10.1542/peds.2013-2936

\section{One in four non-adherent to blood pressure control}

A quarter of patients prescribed antihypertensive medications were found to be either partially or completely non-adherent to their therapy, according to English research published in Heart. Using high-performance liquid chromatographytandem mass spectrometry urine analysis, the authors screened 208 hypertensive patients for 40 commonly prescribed antihypertensive medications in spot urine samples. They reported a clear association between the degree of non-adherence (measured as the numerical difference or the ratio between biochemically detected and prescribed medications) and recorded blood pressure. One-quarter of patients referred for renal denervation were assessed to be completely nonadherent. An accompanying editorial said the authors had answered not only whether patients were compliant but also how to "cheaply find out". "No longer need we guess whether patients have resistant hypertension. We can know."

Heart 2014: 2 April (online). doi: 10.1136/heartjnl-2013-305063; doi: 10.1136/heartjnl-2014-305540

\section{Atopic dermatitis a lifelong illness}

Atopic dermatitis (AD) is likely a lifelong illness with periods of "waxing and waning" skin problems, according to research published in JAMA Dermatology. Data from the Pediatric Eczema Elective Registry (PEER), a 10-year registry with 22550 person-years of observations, including 2416 patients with at least 5 years' follow-up, showed that the odds of a child having no AD symptoms while not using AD medications increased by $30 \%$ with each 6 -monthly survey. However, the proportion of patients who were symptom-free in the past 6 months was low for all ages - about $6 \%$ at age 5 years, about $12 \%$ at age 10 years and about $17 \%$ at age 20 years. "These findings challenge the conventional dogma that most childhood AD 'burns out'", said an accompanying editorial. "Even mild to

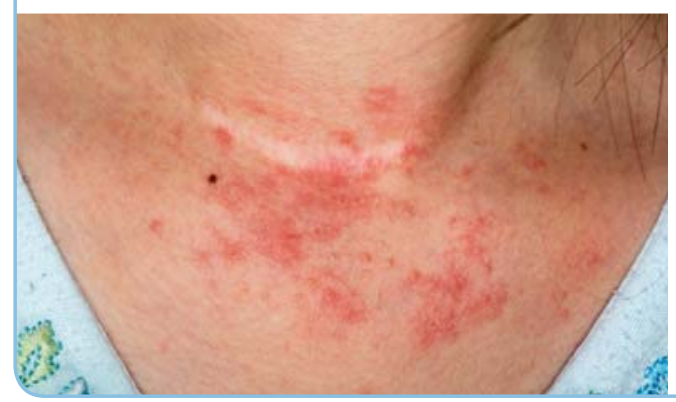
moderate eczema is more likely to persist into adulthood than we previously realized."

JAMA Dermatol 2014; 2 April (online) doi: 10.1001/jamadermatol.2013.10271; doi: 10.1001/jamadermatol.2013.10267

Cate Swannell doi: 10.5694/mjal4.n0421
The MJA's trial of the "Comments" section is coming to an end, but there's still time to send us your short comments on any current health care issue, or let us know if you'd prefer to see an increased News section instead.

\section{This issue's suggested topic:

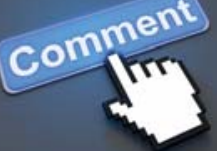 \\ Should health funds cover overseas medical procedures? \\ Yes $\cdot \square$ Depends $\cdot \square$ No}

Email us a brief comment (no more than 100 words) on the above question, or any healthrelated subject. Email your comments to: comments@mja.com.au

Previous issue's suggested topic:

Do you support the proposal to let pharmacists administer vaccines?

I graduated with a Bachelor of Pharmacy in 1994 when "forward pharmacy" was the new thing in patient care. Then, I believed my clinical skills were adequate to provide blood pressure measurements and general medical advice to patients. It was not until I graduated from medicine in 2001 and attained Fellowship of the RACGP that I realised how dangerous I could have been as a pharmacist trying to manage medical illnesses. The keywords are "differential diagnosis" - medical training is what gives a doctor these skills. I don't believe pharmacists are trained to the same degree of skill in diagnosing and treating.

Dr Warwick Marks, General Practitioner, Qld.

When sending your comment, please include your full name, discipline and state of residence, and disclose any relevant information or affiliations that may affect interpretation of your comments.

Visit: https://www.mja.com.au/journal/mja-instructions-authorstypes-articles-published-mja\#Comments

\section{MJA InSight poll! Total respondents: 146}

Is being caring and compassionate with patients as important in medicine as excellent clinical care?
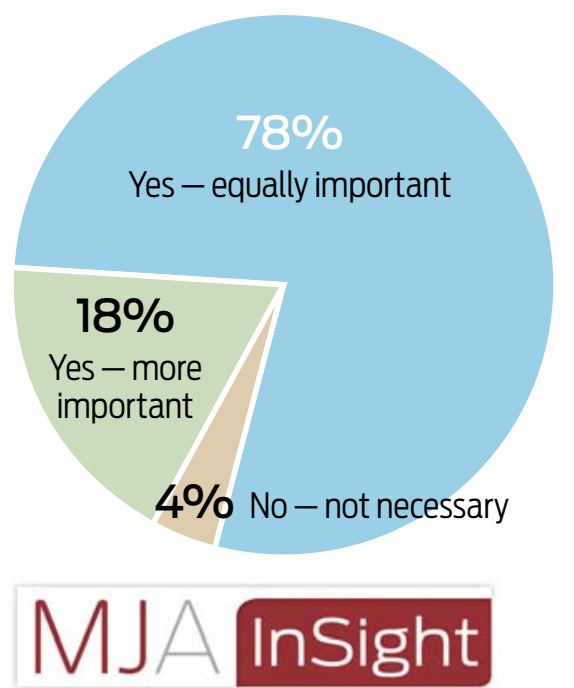

Take part in next week's poll on: www.mja.com.au/insight 